\title{
A new Mathematical Programming Model for the Green Vehicle Routing Problem
}

\author{
Maurizio Bruglieri ${ }^{1}$ \\ Dipartimento di Design, Politecnico di Milano, Milano, Italy \\ Simona Mancini ${ }^{2}$
}

Dipartimento di Matematica e Informatica, Universitá di Cagliari, Cagliari, Italy

Ferdinando Pezzella ${ }^{3}$

DII, Universitá Politecnica delle Marche, Ancona, Italy

Ornella Pisacane ${ }^{4}$

Facoltá di Ingegneria, Universitá degli Studi e-Campus, Novedrate (Como), Italy

\begin{abstract}
A new MILP formulation for the Green Vehicle Routing Problem is introduced where the visits to the Alternative Fuel Stations (AFSs) are only implicitly considered. The number of variables is also reduced by pre-computing for each couple of customers an efficient set of AFSs, only given by those that may be actually used in an optimal solution. Numerical experiments on benchmark instances show that our model outperforms the previous ones proposed in the literature.
\end{abstract}

Keywords: Vehicle Routing, Refueling, Alternative Fuel Vehicles, Efficient set of Alternative Fuel Stations, Mixed Integer Programming 


\section{Introduction}

In the Vehicle Routing Problem (VRP), fuel autonomy is usually assumed sufficient to serve customers in every route. However, with Alternative Fuel Vehicles (AFVs), refuels along the route are needed. Moreover, since Alternative Fuel Stations (AFSs) are not widespread on road networks, refueling stops should be a priori planned to prevent drivers to remain stuck along their routes. The Green VRP (G-VRP) [1] consists in serving a set of customers with a fleet of $m$ AFVs that leave fully refueled from a single depot and can be refueled at AFSs, along their routes. The objective is to minimize the total travel distance. The refueling time is fixed. A fuel consumption rate $(r)$ is given and tanks are totally replenished at AFSs. A maximum route duration $\left(T_{\text {max }}\right)$ is imposed. The G-VRP is modeled in [1] including dummy copies of the AFSs to manage multiple visits at the same AFS. Hereafter we refer to such a formulation as the EMH model. A further formulation in which the AFSs are implicitly addressed is proposed in [2], together with a Branchand-Cut method (hereafter, KK-B\&C). In this paper, the G-VRP is modeled by Mixed Integer Linear Programming (MILP) without cloning AFSs, since this increases the number of nodes and, consequently, the problem complexity. The number of variables employed is also reduced by pre-computing for each couple of customers an efficient set of AFSs, including only those that may be actually used in an optimal solution. Our formulation is tested on two sets of benchmark instances taken by [1], showing that it outperforms both the EMH model and the KK-B\&C.

\section{A New MILP Model for the G-VRP}

The G-VRP is defined on a directed complete graph $G=(N, A)$, where $N=$ $I \cup\{0\}$, with $I$ set of customers and 0 the depot, and $A=\{(i, j): i \in N, j \in$ $N, i \neq j\}$. The set $F$ of available AFSs is known. The following data are given: $\forall i \in N \cup F, \forall j \in N \cup F, i \neq j$, travel time, $t_{i j}$, and travel distance, $d_{i j}$; $\forall i \in I, p_{i}$ is the service time, while $\forall s \in F$ it represents the refueling time; $Q$ is the refueling capacity. Our model is based on the computation of the sets $L_{i j}$ of AFSs that may be convenient for an AFV to move in a feasible way from $i$ to $j, \forall(i, j) \in A$. These sets are computed in the following way. Let

1 Email:maurizio.bruglieri@polimi.it

2 Email:simona.mancini@unica.it

3 Email:pezzella@dii.univpm.it

4 Email:ornella.pisacane@uniecampus.it 
$s^{*}=\arg \min _{s \in F: d_{i s} \leq \frac{Q}{r}, d_{s j} \leq \frac{Q}{r}}\left\{d_{i s}+d_{s j}\right\}$. At the beginning $L_{i j}:=F$ and for each $s \in F$ if $d_{i s}>d_{i s^{*}}$ and $d_{s j}>d_{s^{*} j}$ or $d_{i s}>\frac{Q}{r}$ or $d_{s j}>\frac{Q}{r}$, the AFS $s$ is removed from $L_{i j}$. Moreover, $\hat{t}_{i j s}=t_{i s}+t_{s j}-t_{i j}$ is the detour time of an AFV to visit $s \in L_{i j}$ going from $i$ to $j$ and $\hat{d}_{i j s}=d_{i s}+d_{s j}-d_{i j}$ is its detour distance. We model the G-VRP through the following binary variables: routing variables $x_{i j}, \forall(i, j) \in A$, equal to 1 if node $j$ is visited just after node $i$ (directly or through an AFS), 0 otherwise; $z_{i j s}, \forall(i, j) \in A, \forall s \in L_{i j}$ equal to 1 if AFS $s$ is employed to go from $i$ to $j, 0$ otherwise. Moreover, we use the following continuous variables $\forall i \in N$ : the residual fuel level $y_{i}$ of AFV at $i$; the time $\tau_{i}$ on which $i$ is reached. The MILP model is detailed in the following:

$$
\min \sum_{(i, j) \in A}\left(d_{i j} x_{i j}+\sum_{s \in L_{i j}} \hat{d}_{i j s} z_{i j s}\right)
$$

$$
\begin{gathered}
\sum_{s \in L_{i j}} z_{i j s} \leq x_{i j} \quad \forall(i, j) \in A \\
\sum_{j \in N: j \neq i} x_{i j}=1 \quad \forall i \in I \\
\sum_{i \in N: i \neq j} x_{j i}=\sum_{i \in N: i \neq j} x_{i j} \quad \forall j \in N \\
\sum_{j \in N: j \neq 0} x_{0 j} \leq m \\
\sum_{j \in N: j \neq 0} x_{j 0} \leq m \\
\tau_{j} \geq \tau_{i}+\left(t_{i j}+p_{i}\right) x_{i j}+\sum_{s \in L_{i j}}\left(\hat{t}_{i j s}+p_{s}\right) z_{i j s}-l_{0}\left(1-x_{i j}\right) \forall i \in N, \forall j \in I, i \neq j \\
\tau_{j} \leq T_{m a x}-\left(t_{j 0}+p_{j}\right)-\sum_{s \in L_{j 0}}\left(\hat{t}_{j 0 s}+p_{s}\right) z_{j 0 s} \quad \forall j \in N \backslash\{0\} \\
y_{j} \leq \sum_{s \in L_{i j}}\left(Q-r \cdot d_{s j}\right) z_{i j s}+Q\left(1-\sum_{s \in L_{i j}} z_{i j s}\right) \forall j \in I, \forall i \in I, i \neq j \\
y_{j} \leq y_{i}-r \cdot d_{i j}+2 Q\left(1-x_{i j}+\sum_{s \in L_{i j}} z_{i j s}\right) \quad \forall j \in I, \forall i \in N, i \neq j \\
y_{i} \geq r \cdot d_{i 0}\left(x_{i 0}-\sum_{s \in L_{i j}} z_{i 0 s}\right) \quad \forall i \in I \\
\sum_{s \in L_{i j}} r \cdot d_{s 0} z_{i 0 s} \leq Q \quad \forall i \in I \\
y_{i} \geq \sum_{s \in L_{i j}}\left(r \cdot d_{i s} z_{i j s}\right) \quad \forall(i, j) \in A \\
y_{0} \leq Q \\
x_{i j} \in\{0,1\} \quad \forall(i, j) \in A \\
y_{i} \geq 0, \tau_{i} \geq 0 \quad \forall i \in N \\
z_{i j s} \in\{0,1\} \quad \forall(i, j) \in A, \forall s \in L_{i j}
\end{gathered}
$$

Fig. 1. New formulation for the G-VRP.

Objective function (1) minimizes the total travel distance. Between each 
pair of customers, at most one AFS may be visited (2) while each customer must be visited exactly once (3). Route continuity is assured by (4). The number of AFVs is limited by (5)-(6). Arrival time at each node is ruled by (7) that also exclude sub-tours. Maximum route duration is enforced by (8). Fuel level, at each node, is ruled by (9)-(10). An AFV, after visiting its last customer, must have enough fuel to return to the depot either without refueling (11) or refueling (12). In case of refueling, (13) guarantee the AFV can reach the selected AFS. The AFVs leave fully recharged from the depot thanks to (14). Finally, the variables nature is specified in (15)-(16)-(17).

\section{Some Numerical Results}

We tested the performances of our model on the benchmark instance sets S1 and S3 of [1], each one with 10 instances and every instance with 20 customers, on average. Both our model and EMH one are solved with CPLEX12.5 with a CPU time limit of 3,600 s. In the EMH model, for each AFS, $m$ copies are introduced. Concerning S1, our model optimally solves 7 instances with an average CPU time of 1,772 s and an average Relative MIP Gap (RMG) of $3.22 \%$. Instead, EMH always reaches the CPU time limit (never certifying the optimality) with an average RMG of $29.97 \%$ and, for one instance, it is not able even to find a feasible solution. For three instances, it finds the same optimal value of ours, without certifying its optimality, thus showing that their lower bound is too weak. Comparing our results with those of KK-B\&C (Table 5 of [2]), despite they use a more powerful computer, we detect two more optimal solutions with a lower average CPU time (theirs is 2,373 s) and with a lower average RMG (theirs is $3.5 \%$ ). About S3, our model performs better than on S1, probably because the greater number of AFSs allows exploiting better the variables saving given by the non-generation of dummy copies of the AFSs. Indeed, our model solves to the optimality 9 over 10 instances with an average CPU time of $1,040 \mathrm{~s}$ and an average RMG of $0.68 \%$. While, EMH always reaches the $\mathrm{CPU}$ time limit with an average RMG of $22.42 \%$. On five instances it is not able even to find a feasible solution within the time limit and only on two instances, the optimal value is found. Comparing our results with those of the KK-B\&C, two more optimal solutions are found, with a lower average CPU time (theirs is 1,204 s) and average RMG (theirs is $1.38 \%$ ).

\section{References}

[1] Erdogan, S., and E. Miller-Hooks, A Green Vehicle Routing Problem, Transportation Research Part E. 48 (2012), 100-114.

[2] Koç, C. and I., Karaoglan, The green vehicle routing problem: A heuristic based exact solution approach, Applied Soft Computing. 39 (2016), 154-164. 